\title{
Circadian Liver Metabolism Affects Management of Type 2 Diabetes Mellitus During Cytokine Storm Due to COVID-19
}

\author{
Dipak Kumar ${ }^{1}$ and Sadaf Jahan² \\ 'Zoology Department, Kumar Kalika Memorial College, Jamui, Munger University, Munger, India \\ 2Department of Medical Laboratory Sciences, College of Applied Medical Sciences, Majmaah University, Al-Majmaah, Saudi Arabia
}

\begin{abstract}
Diabetes is managed to keep the blood sugar in normal range. This involves liver as glucose metabolizing organ and sensitization of somatic cells to utilize this glucose for daily energy requirements. The management is subjected to the rhythmic glucose intake as diet and liver circadian cycles that runs parallel to this zeitgeber. COVID-19 patients having diabetes as comorbid condition face the challenges of inflammatory cytokine management along with the organization of glucose. Increased blood glucose level during the cytokine storm further aggravates the pathophysiology of COVID-19 patients leading to high morbidity and mortality in such patients. Clinical treatment of these patients requires multidimensional approach involving circadian variation of hepatic physiology, glucose intake, and inflammatory cytokine release.
\end{abstract}

Keywords: Circadian rhythm; Diabetes; Cytokine storm; Glucose metabolism; COVID-19

Received: October 10, 2021 Revised: October 26, 2021 Accepted: October 30, 2021

Corresponding author: Dipak Kumar, PhD, Zoology Department, KKM College, Jamui, Munger University, Munger, India.

Tel: 91-7417391312, E-mail: thepaque@gmail.com

(a) This is an Open Access article distributed under the terms of the Creative Commons Attribution Non-Commercial License (https://creativecommons.org/licenses/bync/4.0) which permits unrestricted non-commercial use, distribution, and reproduction in any medium, provided the original work is properly cited.

\section{INTRODUCTION}

Human circadian clock comprises of billions of cellular clocks controlled by superchiasmatic nucleus (SCN) present in the hypothalamus as central clock [1]. The cellular clocks show rhythmic variations in their physiology in harmony with the SCN and are present in peripheral tissues including liver, kidney, heart, muscles, peripheral blood leucocytes, etc. [2]. The master clock, $\mathrm{SCN}$, is in the brain which is entrained by light-dark environmental cycle and orchestrates peripheral clocks present in different cells of the body through several pathways such as neuronal, endocrine and blood-borne signals using mechanisms of transcriptional and post transcriptional regulations [3]. The biological rhythm is generated by the core transcriptional and translational feedback loop composed of a number of clock genes brain and muscle ARNT-like (BMAL)1, circadian locomotor output cycles Kaput (CLOCK), period (PER)1, PER2, cryptochrome (CRY)1, CRY2, etc. [4]. Heterodimer formed by the BMAL1, and CLOCK proteins activates transcription of PER, CRY, and other clock regulated genes [5]. Subsequently, PER and CRY proteins are phos- phorylated by either $\varepsilon$ or $\delta$ variant of casein kinase I (CKI) and translocated into the nucleus to repress the transcription of BMAL and CLOCK proteins [6]. Further, a feedback loop made up of RAR related orphan receptor and REV-ERB (aka nuclear receptor subfamily 1 group D member 1, NR1D1) family members reinforce the controls over Bmall gene expression which in turn are positively controlled by CLOCK and BMAL1 proteins [7]. Additionally, the circadian rhythm is fine-tuned by diverse post transcriptional regulations in an organ specific manner.

In liver, food intake is the principal circadian timing zeitgeber which is physiologically relayed to the circadian clock via metabolites and nutrients such as nicotinamide adenine dinucleotide (NAD), polyamines, and glucose [8]. The liver is independent enough to fully uncouple its clock from the central pacemaker as its entrainment is mostly driven by the feeding-fasting cycle [9] Notably, there are metabolic pathways forming distinct feedback loop coupled to the master circadian oscillator which can function primarily intracellularly such as $\mathrm{NAD}^{+} / \mathrm{NADH}$ or impart its systemic consequences on widely distributed peripheral clocks as done by glucose $[10,11]$. The signal at energy and metabolite lev- 
el is relayed to the molecular oscillator through physiological sensors like SIRT protein, PARP-1, AMPK, and PGC-1 $\alpha$ [12]. Moreover, universal signaling molecules like cAMP show circadian rhythm playing its role both as clock component and control output. Recently, a link has been established between clock and Sirtuins under the influence of redox potential. Further, insulin sensitivity has been noted to improve when the Sirtuin 1 expression is upregulated contrary to the cells showing high insulin resistance when Sirtuin 1 expression is downregulated [13]. Moreover, SIRT1 also affects the activity of PGC- $1 \alpha /$ ERR- $\alpha$ complex by deacetylation, which are critical metabolic controlling transcription factors [14].

Not only the liver physiology is subjected to the circadian rhythm under the influence of glucose availability as discussed above, but immune response also shows oscillatory behavior $[15,16]$. Hepatobiliary system must control the number of inflammatory cytokines, vasoactive agents and eicosanoids from the plasma in systemic circulation through detoxification and inactivation processes. Thereby, acting as an important part of systemic and pulmonary host defense and provide protection to the lungs and other organs against injury. Otherwise, increased level of cytokines such as IL-8, IL-1 $1 \beta$, TNF- $\alpha$, MCP-1, MIP- $1 \alpha$ shows deleterious effect on alveolar epithelium and endothelial cells [17]. This can also lead to the local innate immune response and enhance platelets aggregation in lungs rendering diffuse alveolar damage [18]. Alteration in alveolar endothelial and epithelial cell physiology and thereby functions enhance barrier permeability leading to alveolar edema and pulmonary failure. Severe manifestation of fever and pneumonia results into acute respiratory distress syndrome (ARDS) seen in about 20\% of COVID-19 cases [19]. The immunological consequences of SARS-CoV-2 infection (pneumonia) on liver physiology is plausible, but the limiting effects of oxygen and glucose availability for cellular energetics cannot be ignored. Under the ischemic and hypoxic conditions, glucose consumption, lipid accumulation, and depletion of adenosine triphosphate (ATP) lead to the inhibition of cell survival signalling pathways causing hepatocyte death. Additionally, the hypoxic conditions arising from the ARDS and drug-induced liver injury initiate oxidative stress responses causing continuous increase in reactive oxygen species (ROS) [20]. ROS together with their peroxidation products activate transcription factors sensitive to the redox conditions, initiating the release of various proinflammatory factors. Such pathophysiological changes can further aggravate the hypoxic and ischemic parameters leading to the release of toxic metabolites injuring the liver severely. Hence, a crosstalk is established among liver (patho)physiology, lungs pathophysiology, and circadian clock during pulmonary inflammation in diabetic individuals. In this review, we are going to discuss this complex scenario of multifactorial management standing in front of clinical administrations.

\section{DIURNAL RHYTHMS AND GLUCOSE METABOLISM IN LIVER}

The hormone regulating blood glucose concentrations fluctuates with circadian rhythm, expecting the recurrent changes in availability of glucose in the blood stream and its expenditure for energy as a result of changes in external environment [21]. Involved in energy balance, insulin is one of the most important hormone which is secreted during hyperglycaemia exhibits circadian rhythm having more production during the dusk than the dawn [22]. The response of metabolic tissues against the insulin level also shows circadian variation being less insulin sensitive during the evening, as a result circadian variation in blood glucose is seen, and hence evenings have high blood glucose concentrations [23]. Moreover, the peripheral oscillators may show circadian rhythm in gene expression independent to the $\mathrm{SCN}$ producing endogenous rhythm in peripheral tissues as in liver [24]. Temporal variation in peripheral tissue gene expression implicate its functions in lipid and glucose metabolism being carried up in adipose, hepatocyte, cardiac, and skeletal muscle cells.

Studies on murine have demonstrated the significance of circadian clock organisation in the maintenance of glucose homeostasis as well as insulin action [25]. Various genetic models of mouse with mutations in peripheral tissue as well as whole body have revealed important roles for every clock genes. The CLOCK mutant mice show significantly diminished diurnal feeding and are hypophagic and obese as compared with the wild type mice. They exhibit adipocyte atrophy, hepatocyte enlargement with high lipid and glycogen build-up along with hypo-insulinemia, hypercholesterolemia, hyperglycaemia, and hypertriglyceridemia [26]. The glucose induced insulin secretion is observed to be reduced in pancreatic islet showing impaired glucose tolerance in the CLOCK mutant mice. Apart from this, the CRY KO mice exhibit increased expression of genes responsible for the hepatic gluconeogenesis and thereby increases its blood glucose concentrations [27]. Liver specific elimination of BMAL1 in the mice leads to the loss of rhythmic hepatic gene expression responsible for the glucose homeostasis and become hypoglycaemic during their fasting period [28].

As a relationship has been established between diabetes and the clock genes polymorphism in murine studies, epidemiological studies on human have established an association between the clock gene polymorphism and diabetes, and have demonstrated that the circadian disturbances alter the clock gene expression. As expected, the variation in CRY2 gene expression has been found to be related with higher blood glucose levels in non-diabetic individuals and PER2 polymorphisms are seen to be associated with increased blood glucose during the fasting [29]. Additionally, the islets from type 2 diabetics (T2D) and non-diabetics donors were compared, which revealed lower mRNA concentrations of CRY2, PER2, and PER3 genes in individuals without diabetes [30]. The mRNA level further found to be positively correlated with insulin amount, and PER3 and CRY2 expression negatively correlat- 
ed with the glycated haemoglobin in blood. Noticeably, BMAL1, PER3, PPARD, and CRY2 genes expression were found to be altered in women with gestational diabetes as compared to the pregnant normoglycemic women [31].

\section{PERIODIC DRUG METABOLISM IN LIVER}

Detoxifying process of xenobiotic is a multi-step process involving three groups of proteins, which perform different and continuous actions. Phase I proteins use the hydroxylase, oxidase, and reductase activities of the microsomal P450 cytochrome (CYP P450) family of enzymes to functionalize drugs (possibly for inhibition or activation) [32]. In order to increase solubility, phase II proteins bind drugs to hydrophilic molecules. They contribute to the hydrophilization of lipophilic substances so that they are more easily excreted in bile, urine, and faecal material. Sulfotransferase, UDP-glucuronyl transferase, glutathione S-transferase, and Nacetyltransferase are enzymes that perform these processes. Finally, exogenous substances are transported outside the cell through phase III transporters, mainly ABC transporters [33]. Additionally other proteins affects the activity of mostly phase I enzymes in oxidoreductase P450 family along with those three enzymes. It is well known that aminolevulinic acid synthase (ALAS1) is the ratelimiting enzyme for heme constructions and heme is the prosthetic group of all CYP P450 enzymes and requisite for the synthesis of CYP P450 [34]. Furthermore, CYP P450 catalyses the monooxygenase reaction which is useful to extricate the electrons $\left(\mathrm{e}^{-}\right)$ from $\mathrm{NAD}(\mathrm{P}) \mathrm{H}$ and facilitates the shifting of $\mathrm{e}^{-}$to the heme group via the flavin group of CYP P450 oxido-reductase enzyme [35]. To facilitate the effective liver detoxification, it is important to harmonize all proteins, which is key factor for pharmacokinetics. This is feasible via genes regulation which are involved in transcriptional process. Those genes are specific for cell types, day dependent, and provoked by xenobiotics [36]. The xenobiotic receptors, a well-known transcriptional factors, play significant role in the induction of transcription [37].

The nuclear receptors constitutive androstane receptor (CAR), pregnane $\mathrm{X}$ receptor (PXR), and the PAS-domain helix-loop-helix transcription factor aryl hydrocarbon receptor (AhR) are three primary xenobiotic receptors [38]. Chaperone protein and these xenobiotic receptors are coupled in the cell cytoplasm and are mostly present in hepatic tissue and colon region specifically small intestine. These proteins concentrate in the nucleus in response to xenobiotics, where they trigger transcription of phase I, II, and III genes, either directly or by signal transduction [39]. The genes involved in the coding of enzyme also facilitates the process of xenobiotic detoxification which mark up a significant portion of the rhythmically expressed genes in mouse liver, according to a historical transcriptome analysis [40].

Recent research reveals that these genes are controlled by circadian clock-controlled transcription factors rather than being direct targets of BMAL [41]. The PARbZip transcription factors play a critical part in this control, according to studies in genetically modified mice [42]. Deletion studies underline their importance in this way that mice lacking these three genes are generated with a standard mendelian proportion with no obvious abnormalities, although the survival is found to be only up to $20 \%$ in one year. Broad decrease in the expression of genes coding for enzymes are reported in the PARbZip-deficient mice. As per gene expression analysis, these enzymes are involved in the kidney and liver for detoxification of xenobiotic with all effective phases [43]. Some of these enzymes, such as Cyp3a4 and Mdrla (MultiDrug Resistance 1a or Abcb1a), are likely regulated by PARbZip proteins through rhythmic binding to their promoters [44]. However, PARbZip transcription factors also influence expression of CAR, which is significantly reduced in the liver and small intestine of PARbZip defective mice with a slowed rhythm. As a result, for the whole day phase I, II, and III enzyme induction is found to be least in these animals [40]. Thus, in PARbZip-deficient animals, the time-dependent activation of Cyp2b10 mRNA by phenobarbital is severely hampered. Moreover, vulnerability of the mice are found against mitoxantrone and cyclophosphamide, the chemotherapy medicines. Mice lacking the clock genes Clock and Bmall share this behaviour, as these mice also have downregulation of PARbZip transcription factors [45]. It is worth noting that Drosophila's timedependent pesticide toxicity is mediated by comparable processes, including the control of the CAR ortholog DHR96 by the PARbZip ortholog Pdp1 and the rhythmic expression of phase II enzymes [46]. Therefore, in rhythmic drug detoxification other clock-regulated genes are also intricated, notwithstanding the relevance of PARbZip transcription factor in circadian pharmacokinetics [47].

Retinoid-related orphan receptors (ROR) and ROR-KO mice, for example, have a defect in the expression of various phase I and phase II enzymes, most likely as a result of the CAR xenobiotic receptor's expression being disrupted [48]. A functioning circadian clock is required for this time-dependent activation of Cypla1. Finally, acetaminophen time-dependent toxicity appears to be caused by rhythmic CYP2E1 expression, which is caused by CRY1's rhythmic suppression of the HNF1 nuclear receptor on the Cyp2e1 promoter [49]. While the relative impact of each of these systems on global rhythmic drug detoxification in the mouse liver has yet to be established, there is no doubt that the circadian clock is a prominent player in this field [40]. A recent study found that an intact circadian clock in the liver is required for daily cycles in acetaminophen toxicity and pentobarbital sleep time, implying that the liver clock governs some elements of drug metabolism. Using wide expression data of genome from multiple tissues, researchers discovered that $43 \%$ of all genes, including many therapeutic targets, had daily expression oscillations in at least one organ. As a result of harmony in the availability of the medication and its metabolites, as well as in their target expressions, the outcome of therapeutic drugs may be affected by time of day [50].

Metformin (also known as Glucophage) is the effective medicine against $\mathrm{T} 2 \mathrm{D}$ in the world. It is also prescribed for the treatment of polycystic ovarian syndrome [51]. Furthermore, it is also under research against and perhaps a way to slow down the aging 
process [52]. It is thought to work in the body by blocking mitochondrial complex I. The cellular ATP synthesis is decreased by reduced flux through the electron transport chain, which promotes AMP-activated protein kinase activation (AMPK) [53]. In obese mice, liver-specific deletion of the upstream activation kinase LKB1 (expressed by the Stk11 gene) of AMPK eliminated the metformin-induced reduction in fasting blood glucose, which means that metformin-mediated changes in liver glucose require the production of AMPK phosphorylation catalysed by LKB1 [54]. On the other hand, metformin can reduce blood glucose synthesis in liver cells of mice with liver-specific AMPK1 and AMPK2 deficiency. This indicates that AMPK is not required for hepatic glucose production with the treatment of metformin [55]. In addition, a recent study found that the intracellular magnesium concentration fluctuates daily in many species, which may change kinetics in many signal transduction pathways, including AMPK activation [56]. Because the effect of chronic low-dose metformin on liver fatty acid accumulation and liver glucose production seems to depend on AMPK-dependent phosphorylation of acetyl-CoA carboxylase (ACC) [57]. These data suggest that clinical efficacy of metformin is affected by the timing. Recently, it was found that co-treatment with melatonin can improve the effect of metformin in improving glucose tolerance, confirming the hypothesis that the biological clock affects the response of metformin [58].

COVID-19 infected patient usually shows the decrease in drug metabolism due to supressed CYP expressions. It is difficult to say how rapidly CYP expression and metabolism get inhibited following the initial SARS-CoV-2 infection, or how long the suppression sustain, based on the current data. The laboratory tests were routinely done 2-5 days after the patient was admitted in trials where cytokines were assessed. Patients are not admitted to the hospital for a COVID-19 infection until they have been infected for an average of 7 days. In vitro experiments using cytokines and other inflammatory proteins have revealed that CYP mRNA is suppressed maximally within 24 hours after cytokine exposure. As a result, it is likely that patients' CYP expression would be downregulated and their metabolic capacity will be reduced during the early stages of infection. Liu et al. [59] offered valuable insight into the differences in IL-6 levels between severe and mild COVID-19 patients in a longitudinal study. IL-6 levels in severe patients were raised to an average of $70 \mathrm{pg} / \mathrm{mL}, 3$ days after disease onset, and remained elevated until 15 days, when they began to decline. IL-6 does not reach a peak in individuals with mild disease and maintains a steady level of $20 \mathrm{pg} / \mathrm{mL}$ throughout the illness. As a result, individuals are likely to have metabolism problems even before they become symptomatic, are brought to the hospital, or have liver disease diagnosed. Because patients may have compromised metabolic capacity regardless of the onset/ length of COVID-19 or the extent of liver dysfunction. It is possible that, in addition to the cytokine storm, toxic drug levels and increased hepatotoxicity from comorbidity, drugs are contributing to COVID-19 patients' abnormal liver health. Hepatocytes have extraordinary regenerating powers, with the exception of a tiny percentage of people who may have serious liver damage. CYP enzymes, like drug-drug interactions, normally recover fast if the offending compounds are no longer present in the body. In this situation, the principal offending agents, inflammatory cytokines, have been shown to return to normal levels around 15 days after infection onset, allowing the CYPs to recover gradually. After hospitalization, cytokine levels, liver function tests, and plasma for narrow therapeutic index medicines must all be evaluated and examined for the restoration of the CYP-mediated metabolic system [60].

\section{CIRCADIAN IMMUNE REGULATION}

The immune system is a second significant pharmaceutical target with strong circadian modulation [61]. Endotoxic shock susceptibility and diurnal fluctuations in white blood cell count have long been observed. Recent research, on the other hand, demonstrates that cell-autonomous clocks within immune cells control a wide range of circadian immunological characteristics. For example, $\mathrm{T}$ cells respond to stimuli in a circadian manner, while macrophages in turn use their own biological clock to promote immune responses in a similar circadian manner [62]. In contrast, there are lesser reports on fluctuated circadian gene expression and circadian B cell activity which is described in peripheral blood mononuclear cells, and are much smaller than those observed in other tissues such as the liver [63]. In addition, the number of certain immune cells in the circulation (such as monocytes, neutrophils, and lymphocytes) and the levels of cytokines (such as IL-6 and TNF) in plasma have a 24-hour daily rhythm, which gets transformed into an acute infection reaction. The ramifications of widespread circadian control of immune function go far beyond the aforementioned daily variation in infective vulnerability [64]. Circadian fluctuation in variables with agglomeration of platelets and adhesion to actual expression of clotting factors like PAI-1 supports a substantial circadian oscillation of blood clotting [65]. Many immune cells, such as hematopoietic stem cells, are regulated by circadian clocks [50]. Finally, circadian immune modulation causes diurnal fluctuations in immunological variables like inflammation that is a key factor in many diseases.

In terms of cytokine expression, both transcription of cytokineencoding genes and activation of cytokine release are strongly influenced by the time of day. Some cytokines are known to be activated by clock proteins, whereas others are suppressed by them [66]. Clock regulation is found in macrophages and monocytes, as well as T cells, and has an impact on these cells' immunological responses to pathogens [67]. It has been claimed that COVID-19 patients who are mechanically ventilated have type I interferon inhibited responses [68]. It is likely that a misalignment here could influence the likelihood of a cytokine storm forming. For example, $T$ cells respond to stimuli in a circadian manner, while macrophages in turn use their own biological clock to promote immune responses in a similar circadian manner. In addition, the number of certain immune cells in the circulation (such as mono- 
cytes, neutrophils, and lymphocytes) and the plasma levels of cytokines (such as TNF- $\alpha$ and IL-6) have a 24-hour daily rhythm, which is transformed in an acute infection reaction [69].

The multifunctional cytokine IL- 6 is involved in the pathogenesis of T2D. The presence of high levels of IL- 6 in the blood is a strong predictor of $\mathrm{T} 2 \mathrm{D}$ and is thought to play a role in the development of inflammation, insulin resistance, and cellular dysfunction [70]. However, there are many evidences that IL-6 has antiinflammatory properties and improves glucose metabolism. The pleiotropic nature of IL- 6 may be explained by its complicated signal transduction process. Classic signalling and trans-signalling are the two separate signalling mechanisms that IL-6 use. Inflammation rises with age, prompting the term "inflammaging" to be coined to describe the process. One of the reasons why older people are more susceptible to infection is because their present baseline of inflammation is higher [71]. COVID-19 has been linked to a cytokine storm, which causes an increase in pro-inflammatory cytokines (TNF-, IL-1, IL-6, and IL-1), which has been linked to an increase in the elderly [72]. As a result, the elderly are particularly sensitive to COVID-19 conditions. In addition to the overlapping pathophysiology of T2D neurological complications, the potential for blood glucose increases to promote inflammation through interferon regulatory factor 5 (IRF5) activity after SARS-CoV-2 infection in neuronal impaired patients may create a "perfect storm" of excessive immune response. As a result, neuroinflammation, immunosenescence, and neurodegeneration are seen in Alzheimer's patients, leading to microglia malfunction and amyloid-beta accumulation [72], as well as a loss of periph- eral immune response.

Recent research has discovered that abnormal accumulation of metabolites (such as urate crystals or lipid peroxidases), hyperglycaemia/obesity, nucleic acids, or physiologically active substances released by necrotic cells can all cause inflammation [73]. Pathogen associated molecular patterns are external stimuli associated with pathogens, and danger-associated molecular patterns are endogenous chemicals emitted from necrotic cells [74]. Inflammation caused by endogenous factors is called aseptic inflammation, because it occurs without infection by pathogenic microorganisms [75]. In addition, the discovery of inflammasomes has promoted our understanding of the underlying mechanisms of many previously unknown diseases [76]. These diseases are considered as new disease entities, known as auto-inflammatory syndromes. The development of diseases such as arteriosclerosis, heart failure, $\mathrm{T} 2 \mathrm{D}$, and cancer is the outcome of abnormal inflammasome conditions [77].

\section{SUMMARY}

Liver is the principal metabolic organ controlling blood glucose level which is at the centre of tight interplay among metabolism homeostasis, circadian rhythm and immune responses. Interpreting several studies, it has been established that the multifaceted clock-dependent management by negative and positive regulators of central clock machinery with several clock-input-output pathways converge on rate limiting molecules of the glucose metabolism [78]. On the other hand, several observations are made

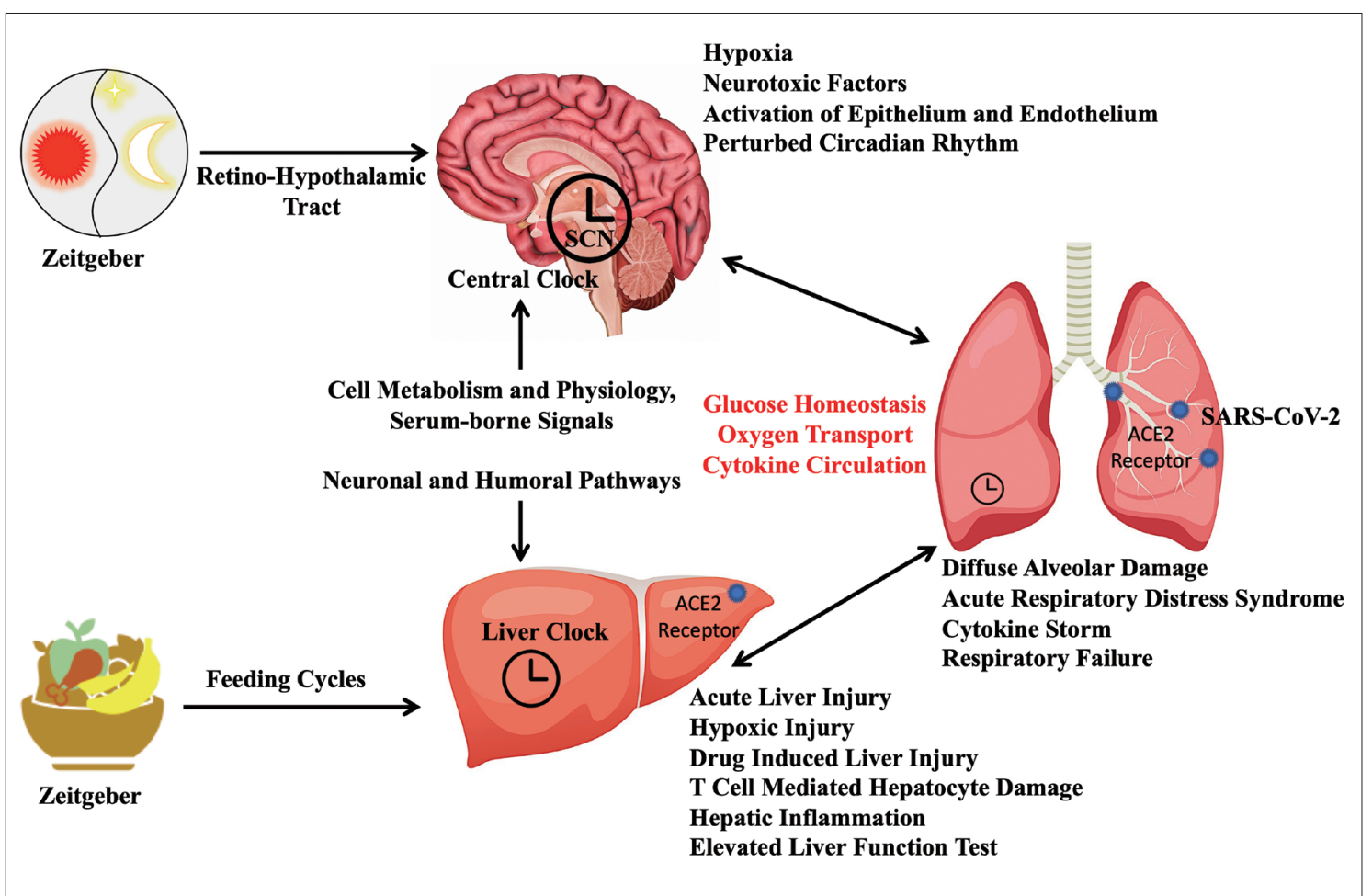

Figure 1. The interplay among brain bearing master circadian clock, liver as a site of xenobiotic metabolism and glucose homeostasis, and lungs responsible for oxygen availability and initiation of inflammatory response after SARS-CoV-2 infection has been depicted. SCN, superchiasmatic nucleus. 
validating that the cell-autonomous clocks within immune cells control a wide range of circadian immunological attributes. For instances, CLOCK/BMAL1 driven transcription factors control the expression of gluconeogenic and glycolytic genes, and CRY protein regulates gluconeogenesis in liver by employing the alternative mechanisms through interaction either with glucocorticoid receptor or G protein-coupled receptors. Notably, liver specific loss of clock function has been seen to lead high blood-glucoselevel fluctuations [22].

Based on recent observations on crosstalk between viral infection and circadian clock, it seems that we are highly vulnerable during early hours of dawn to some of the respiratory viruses. This can be attributed to the circadian fluctuations of immunity parameters. It is now empirical to determine whether SARS-COV-2 show such opportunistic behaviour. A diabetic individual infected with SARS-COV-2 faces simultaneous challenges of maintaining insulin sensitivity and inflammatory response. Various immune cells such as monocyte, neutrophils, and lymphocytes in systemic circulation as well as plasma levels of different cytokines such as TNF- $\alpha$ and IL-6 show rhythmic variations during the about 24-hour period. Immune functions such as leukocyte homing, and innate immunity responses also show circadian variations along with the components of immune system. Supporting the fact, it has been observed that IL- 1 and IL- 6 are only detectable during 12:00-3:00 am in the serum of healthy individuals [79]. Such rhythms have been equally seen in both males and females. On the other hand, plasma level of IFN- $\gamma$ was highest during the period of 12:00-3:00 am and lowest during 8:00-11:00 am. Also, the cytokines such as IL-10 and IL-12 show the diurnal rhythmicity [80]. The rhythmic period of IFN- $\gamma$ and IL-12 has been found to be synchronous with each other and a harmonious relationship has been found among IL-1, IL-10, and TNF- $\alpha$. COVID-19 patients show exaggerated inflammatory response called cytokine storm where immunological rhythmicity may get disturbed [81].

Taken all these interplays together (Figure 1), there are various aspects of the ongoing COVID-19 pandemic that can be related to the circadian biological rhythms such as blood glucose level, liver physiology in diabetics, inflammatory responses from different organs involved in SARS-COV-2 infection, etc. Although it is biologically feasible, there has been inadequate attention towards the protective potential of circadian rhythm in diabetic individuals suffering from COVID-19. Further, the circadian drug metabolism in liver of diabetic individuals must be taken into consideration when medication is prescribed for the symptomatic relief from the COVID-19. There are reports of complications when glucocorticoids are administered to the diabetic individuals [82]. This can be attributed to the fine-tuned circadian rhythm related with the blood glucose and inflammatory response. Whenever there is a cross talk among glucose metabolism, immunity physiology, and circadian biological rhythms in diseases like COVID-19, a holistic therapeutic approach should be considered even after the ongoing pandemic is over.
Acknowledgments

None

\section{Conflicts of Interest}

The authors have no potential conflicts of interest to disclose.

\section{Author Contributions}

Conceptualization: Dipak Kumar. Investigation: all authors. Visualization: Dipak Kumar. Writing-original draft: all authors. Writing—review \& editing: all authors.

\section{ORCID iDs}

Dipak Kumar (10)

https://orcid.org/0000-0001-6104-9447

Sadaf Jahan (10)

https://orcid.org/0000-0002-6976-5672

\section{REFERENCES}

1. Buijs RM, Kalsbeek A. Hypothalamic integration of central and peripheral clocks. Nat Rev Neurosci 2001;2:521-526.

2. Dibner C, Schibler U, Albrecht U. The mammalian circadian timing system: organization and coordination of central and peripheral clocks. Annu Rev Physiol 2010;72:517-549.

3. Ishida N, Kaneko M, Allada R. Biological clocks. Proc Natl Acad Sci U S A 1999;96:8819-8820.

4. Cox KH, Takahashi JS. Circadian clock genes and the transcriptional architecture of the clock mechanism. J Mol Endocrinol 2019;63:R93-R102.

5. Kwon I, Lee J, Chang SH, Jung NC, Lee BJ, Son GH, et al. BMAL1 shuttling controls transactivation and degradation of the CLOCK/BMAL1 heterodimer. Mol Cell Biol 2006;26:7318-7330.

6. Narasimamurthy R, Virshup DM. The phosphorylation switch that regulates ticking of the circadian clock. Mol Cell 2021;81:1133-1146.

7. Brenna A, Albrecht U. Phosphorylation and circadian molecular timing. Front Physiol 2020;11:612510.

8. Guan D, Xiong Y, Trinh TM, Xiao Y, Hu W, Jiang C, et al. The hepatocyte clock and feeding control chronophysiology of multiple liver cell types. Science 2020;369:1388-1394.

9. Kinouchi K, Magnan C, Ceglia N, Liu Y, Cervantes M, Pastore N, et al. Fasting imparts a switch to alternative daily pathways in liver and muscle. Cell Rep 2018;25:3299-3314.e6.

10. Stenvers DJ, Scheer FAJL, Schrauwen P, la Fleur SE, Kalsbeek A. Circadian clocks and insulin resistance. Nat Rev Endocrinol 2019;15:75-89.

11. Mason IC, Qian J, Adler GK, Scheer FAJL. Impact of circadian disruption on glucose metabolism: implications for type 2 diabetes. Diabetologia 2020; 63:462-472.

12. Morris AR, Stanton DL, Roman D, Liu AC. Systems level understanding of circadian integration with cell physiology. J Mol Biol 2020;432:3547-3564.

13. Liang F, Kume S, Koya D. SIRT1 and insulin resistance. Nat Rev Endocrinol 2009;5:367-373.

14. Nakahata Y, Kaluzova M, Grimaldi B, Sahar S, Hirayama J, Chen D, et al. The NAD+-dependent deacetylase SIRT1 modulates CLOCK-mediated chromatin remodeling and circadian control. Cell 2008;134:329-340.

15. Curtis AM, Bellet MM, Sassone-Corsi P, O’Neill LA. Circadian clock proteins and immunity. Immunity 2014;40:178-186.

16. Coiffard B, Diallo AB, Mezouar S, Leone M, Mege JL. A tangled threesome: circadian rhythm, body temperature variations, and the immune system. Biology (Basel) 2021;10:65

17. Fara A, Mitrev Z, Rosalia RA, Assas BM. Cytokine storm and COVID-19: a chronicle of pro-inflammatory cytokines. Open Biol 2020;10:200160.

18. Maiese A, Manetti AC, La Russa R, Di Paolo M, Turillazzi E, Frati P, et al. Autopsy findings in COVID-19-related deaths: a literature review. Forensic Sci Med Pathol 2021;17:279-296.

19. Lin SH, Zhao YS, Zhou DX, Zhou FC, Xu F. Coronavirus disease 2019 (CO- 
VID-19): cytokine storms, hyper-inflammatory phenotypes, and acute respiratory distress syndrome. Genes Dis 2020;7:520-527.

20. Nardo AD, Schneeweiss-Gleixner M, Bakail M, Dixon ED, Lax SF, Trauner M. Pathophysiological mechanisms of liver injury in COVID-19. Liver Int 2021;41:20-32.

21. Badar A. Circadian rhythm in health and disease. J Pak Med Assoc 2018; 68:833-834.

22. Shi SQ, Ansari TS, McGuinness OP, Wasserman DH, Johnson CH. Circadian disruption leads to insulin resistance and obesity. Curr Biol 2013;23:372381.

23. Tan E, Scott EM. Circadian rhythms, insulin action, and glucose homeostasis. Curr Opin Clin Nutr Metab Care 2014;17:343-348.

24. Balsalobre A. Clock genes in mammalian peripheral tissues. Cell Tissue Res 2002;309:193-199.

25. Karthikeyan R, Marimuthu G, Spence DW, Pandi-Perumal SR, BaHammam AS, Brown GM, et al. Should we listen to our clock to prevent type 2 diabetes mellitus? Diabetes Res Clin Pract 2014;106:182-190.

26. Ruan W, Yuan X, Eltzschig HK. Circadian rhythm as a therapeutic target. Nat Rev Drug Discov 2021;20:287-307.

27. Liu Z, Qian M, Tang X, Hu W, Sun S, Li G, et al. SIRT7 couples light-driven body temperature cues to hepatic circadian phase coherence and gluconeogenesis. Nat Metab 2019;1:1141-1156.

28. Li J, Lv S, Qiu X, Yu J, Jiang J, Jin Y, et al. BMAL1 functions as a cAMP-responsive coactivator of HDAC5 to regulate hepatic gluconeogenesis. Protein Cell 2018;9:976-980.

29. Škrlec I, Milić J, Cilenšek I, Petrovič D, Wagner J, Peterlin B. Circadian clock genes and myocardial infarction in patients with type 2 diabetes mellitus. Gene 2019;701:98-103.

30. Yu R, Tian L, Ding Y, Gao Y, Li D, Tang Y. Correlation between inflammatory markers and impaired circadian clock gene expression in type 2 diabetes mellitus. Diabetes Res Clin Pract 2019;156:107831.

31. Choudhury S, Tan T, Lazarus K, Meeran K. The use of prednisolone versus dual-release hydrocortisone in the treatment of hypoadrenalism. Endocr Connect 2021;10:R66-R76.

32. Siminszky B. Plant cytochrome P450-mediated herbicide metabolism. Phytochem Rev 2006;5:445-458.

33. Dietrich CG, Geier A, Oude Elferink RP. ABC of oral bioavailability: transporters as gatekeepers in the gut. Gut 2003;52:1788-1795.

34. Peyer AK. Regulation of hepatic heme synthesis by drugs, bile acids and nutrition: a transcriptional network regulating [delta]-aminolevulinic acid synthase 1 (ALAS1) [dissertation]. Basel: University of Basel, 2008.

35. Julsing MK, Cornelissen S, Bühler B, Schmid A. Heme-iron oxygenases: powerful industrial biocatalysts? Curr Opin Chem Biol 2008;12:177-186.

36. Zhao M, Zhao H, Deng J, Guo L, Wu B. Role of the CLOCK protein in liver detoxification. Br J Pharmacol 2019;176:4639-4652.

37. Kar S, Leszczynski J. Recent advances of computational modeling for predicting drug metabolism: a perspective. Curr Drug Metab 2017;18:11061122 .

38. Larigot L, Juricek L, Dairou J, Coumoul X. AhR signaling pathways and regulatory functions. Biochim Open 2018;7:1-9.

39. Mackowiak B, Wang H. Mechanisms of xenobiotic receptor activation: direct vs. indirect. Biochim Biophys Acta 2016;1859:1130-1140.

40. Dallmann R, Brown SA, Gachon F. Chronopharmacology: new insights and therapeutic implications. Annu Rev Pharmacol Toxicol 2014;54:339-361.

41. Trott AJ, Menet JS. Regulation of circadian clock transcriptional output by CLOCK:BMAL1. PLoS Genet 2018;14:e1007156.

42. Gachon F. Physiological function of PARbZip circadian clock-controlled transcription factors. Ann Med 2007;39:562-571.

43. Hansen LG, Shane BS. Xenobiotic metabolism. In: Cockerham LG, Shane BS, editors. Basic environmental toxicology. Boca Raton: CRC Press, 2019, p. 49-105.

44. Weger BD, Gobet C, David FPA, Atger F, Martin E, Phillips NE, et al. Systematic analysis of differential rhythmic liver gene expression mediated by the circadian clock and feeding rhythms. Proc Natl Acad Sci U S A 2021; 118:e2015803118.

45. Ozturk N, Ozturk D, Kavakli IH, Okyar A. Molecular aspects of circadian pharmacology and relevance for cancer chronotherapy. Int J Mol Sci 2017; 18:2168.

46. Gachon F, Firsov D. The role of circadian timing system on drug metabo- lism and detoxification. Expert Opin Drug Metab Toxicol 2011;7:147-158.

47. Green CB, Takahashi JS. Xenobiotic metabolism in the fourth dimension: PARtners in time. Cell Metab 2006;4:3-4.

48. Daujat-Chavanieu M, Gerbal-Chaloin S. Regulation of CAR and PXR expression in health and disease. Cells 2020;9:2395.

49. Sezgin S, Hassan R, Zühlke S, Kuepfer L, Hengstler JG, Spiteller M, et al. Spatio-temporal visualization of the distribution of acetaminophen as well as its metabolites and adducts in mouse livers by MALDI MSI. Arch Toxicol 2018;92:2963-2977.

50. Dierickx P, Van Laake LW, Geijsen N. Circadian clocks: from stem cells to tissue homeostasis and regeneration. EMBO Rep 2018;19:18-28.

51. Wentling G. Glucophage ${ }^{\mathbb{\circledR}}$ (metformin hydrochloride), the wonder drug: a biguanide class treatment of type 2 diabetes. Monarch Rev 2017;4:112-128.

52. Soukas AA, Hao H, Wu L. Metformin as anti-aging therapy: is it for everyone? Trends Endocrinol Metab 2019;30:745-755.

53. Pernicova I, Korbonits M. Metformin--mode of action and clinical implications for diabetes and cancer. Nat Rev Endocrinol 2014;10:143-156.

54. Zaharenko L. Pharmacogenetics of efficiency and tolerance of the peroral antidiabetic drug metformin [dissertation]. Riga: University of Latvia, 2015.

55. Agius L, Ford BE, Chachra SS. The metformin mechanism on gluconeogenesis and AMPK activation: the metabolite perspective. Int J Mol Sci 2020;21: 3240 .

56. Stangherlin A, O’Neill JS. Signal transduction: magnesium manifests as a second messenger. Curr Biol 2018;28:R1403-R1405.

57. Vancura A, Nagar S, Kaur P, Bu P, Bhagwat M, Vancurova I. Reciprocal regulation of AMPK/SNF1 and protein acetylation. Int J Mol Sci 2018;19:3314.

58. Dantas-Ferreira RF, Raingard H, Dumont S, Schuster-Klein C, GuardiolaLemaitre B, Pevet $\mathrm{P}$, et al. Melatonin potentiates the effects of metformin on glucose metabolism and food intake in high-fat-fed rats. Endocrinol Diabetes Metab 2018;1:e00039.

59. Liu X, Zhao J, Wang H, Wang W, Su X, Liao X, et al. Metabolic defects of peripheral T cells in COVID-19 patients. J Immunol 2021;28:ji2100068.

60. Deb S, Arrighi S. Potential effects of COVID-19 on cytochrome P450-mediated drug metabolism and disposition in infected patients. Eur J Drug Metab Pharmacokinet 2021;46:185-203.

61. Scheiermann C, Kunisaki Y, Frenette PS. Circadian control of the immune system. Nat Rev Immunol 2013;13:190-198.

62. Mavroudis PD, Scheff JD, Calvano SE, Androulakis IP. Systems biology of circadian-immune interactions. J Innate Immun 2013;5:153-162.

63. Sukumaran S, Almon RR, DuBois DC, Jusko WJ. Circadian rhythms in gene expression: relationship to physiology, disease, drug disposition and drug action. Adv Drug Deliv Rev 2010;62:904-917.

64. Nakao A. Temporal regulation of cytokines by the circadian clock. J Immunol Res 2014;2014:614529.

65. Budkowska M, Lebiecka A, Marcinowska Z, Woźniak J, Jastrzębska M, Dołęgowska B. The circadian rhythm of selected parameters of the hemostasis system in healthy people. Thromb Res 2019;182:79-88.

66. Hergenhan S, Holtkamp S, Scheiermann C. Molecular interactions between components of the circadian clock and the immune system. J Mol Biol 2020; 432:3700-3713.

67. Cermakian N, Westfall S, Kiessling S. Circadian clocks and inflammation: reciprocal regulation and shared mediators. Arch Immunol Ther Exp (Warsz) 2014;62:303-318.

68. Walz L, Cohen AJ, Rebaza AP, Vanchieri J, Slade MD, Dela Cruz CS, et al. JAK-inhibitor and type I interferon ability to produce favorable clinical outcomes in COVID-19 patients: a systematic review and meta-analysis. BMC Infect Dis 2021;21:47.

69. Suffredini AF, O'Grady NP. Pathophysiological responses to endotoxin in humans. In: Brade H, Opal SM, Vogel SN, Morrison DC, editors. Endotoxin in health and disease. Boca Raton: CRC Press, 2020, p. 817-830.

70. Jalkanen J, Hollmén M, Jalkanen S. Interferon beta-1a for COVID-19: critical importance of the administration route. Crit Care 2020;24:335.

71. Stebbing J, Sánchez Nievas G, Falcone M, Youhanna S, Richardson P, Ottaviani S, et al. JAK inhibition reduces SARS-CoV-2 liver infectivity and modulates inflammatory responses to reduce morbidity and mortality. Sci Adv 2021;7:eabe4724.

72. Kumar D, Jahan S, Khan A, Siddiqui AJ, Redhu NS, Wahajuddin, et al. Neurological manifestation of SARS-CoV-2 induced inflammation and possible therapeutic strategies against COVID-19. Mol Neurobiol 2021;58:3417- 
3434.

73. Glass CK, Olefsky JM. Inflammation and lipid signaling in the etiology of insulin resistance. Cell Metab 2012;15:635-645.

74. Roh JS, Sohn DH. Damage-associated molecular patterns in inflammatory diseases. Immune Netw 2018;18:e27.

75. Siewert E, Bort R, Kluge R, Heinrich PC, Castell J, Jover R. Hepatic cytochrome $\mathrm{P} 450$ down-regulation during aseptic inflammation in the mouse is interleukin 6 dependent. Hepatology 2000;32:49-55.

76. Jahan S, Kumar D, Chaturvedi S, Rashid M, Wahajuddin M, Khan YA, et al. Therapeutic targeting of NLRP3 inflammasomes by natural products and pharmaceuticals: a novel mechanistic approach for inflammatory diseases. Curr Med Chem 2017;24:1645-1670.

77. Sharma A, Tate M, Mathew G, Vince JE, Ritchie RH, de Haan JB. Oxidative stress and NLRP3-inflammasome activity as significant drivers of diabetic cardiovascular complications: therapeutic implications. Front Physiol 2018; 9:114.

78. Anna G, Kannan NN. Post-transcriptional modulators and mediators of the circadian clock. Chronobiol Int 2021;38:1244-1261.

79. Annamneedi VP, Park JW, Lee GS, Kang TJ. Cell autonomous circadian systems and their relation to inflammation. Biomol Ther (Seoul) 2021;29:31-40.

80. Petrovsky N, Harrison LC. The chronobiology of human cytokine production. Int Rev Immunol 1998;16:635-649.

81. Mélo Silva Júnior ML, Souza LMA, Dutra REMC, Valente RGM, Melo TS Review on therapeutic targets for COVID-19: insights from cytokine storm. Postgrad Med J 2021;97:391-398.

82. Deng F, Gao D, Ma X, Guo Y, Wang R, Jiang W, et al. Corticosteroids in diabetes patients infected with COVID-19. Ir J Med Sci 2021;190:29-31. 